\title{
THE OPERATIONAL STATUS OF POHANG LIGHT SOURCE
}

\author{
J. Y. Huang*, T.-Y. Lee, E. S. Park, M. G. Kim, E. S. Kim, J. Choi, S. H. Nam, and M. Yoon, \\ Pohang Accelerator Laboratory, San 31, Hyoja-dong, Pohang 790-784, Korea
}

\begin{abstract}
From the year 2000, the PLS storage ring stores $2.5 \mathrm{GeV}$ high-energy electron beam by raising the energy from the $2.0 \mathrm{GeV}$ injection energy. The stored current is presently $180 \mathrm{~mA}$, which is limited by the available $\mathrm{rf}$ energy at $2.5 \mathrm{GeV}$. In May 2000 , we succeeded in storing $300 \mathrm{~mA}$ at $2.0 \mathrm{GeV}$ achieving the last design goal of PLS. Further, the new record of the maximum beam current, 450mA, was made in April 2001. At present, PLS has 13 beamlines in operation, 1 beamline under commissioning and 6 beamlines under construction. Other progresses and issues of the PLS operation will be discussed further.
\end{abstract}

\section{INTRODUCTION}

Pohang Light Source(PLS) [1] is a $2.0-2.5 \mathrm{GeV}$, lowemittance light source dedicated to users since 1995. Until 1998 , PLS had been run only at $2.0 \mathrm{GeV}$. After the successful demonstration of high energy $(>2.4 \mathrm{GeV})$ beam operation in 1998, the machine was fine tuned further in 1999 to accomodate better the high energy operation. In 2000 , the overall operation energy was $2.5 \mathrm{GeV}$ with the average injection current of $170 \mathrm{~mA}$, which is well above the design value of $150 \mathrm{~mA}$ at $2.5 \mathrm{GeV}$. The maximum injection current is around $180 \mathrm{~mA}$ being limited by total rf power, but all the beam instabilities were suppressed at this energy. At $2.0 \mathrm{GeV}$, we succeeded in storing $300 \mathrm{~mA}$ with a systematic control of the chromaticity and $\mathrm{rf}$ temperature in May 2000 [2], which was the last unachieved design goal of PLS storage ring. Further, the new record of the maximum beam current, $430 \mathrm{~mA}$ was made in October 2000 as shown in Fig.1. From early this year, we maintain a Reference Orbit chosen as a best empirical orbit during the machine-tuning period after the winter shutdown. At present, various machine upgrade projects are ongoing in PLS, such as the precision beam diagnostic station, development of the new control system, cure of the closed orbit drift by thermal effects, increasing the rf power for compensation of the energy loss by IDs, and the new rf control electronics.

There are 13 beam lines in operation including the first insertion device(U7) beam line at PLS. Also an EPU6 for the elliptically polarized beam is under commissioning and a U10 is planned to be istalled in this summer. There was a total of 237 experiments out of 322 proposals submitted in 2000 indicating steady increase of the user participation [3]. In Table 1, total beam-time provided and user service time since 1995 are summarized.

*huang@postech.ac.kr

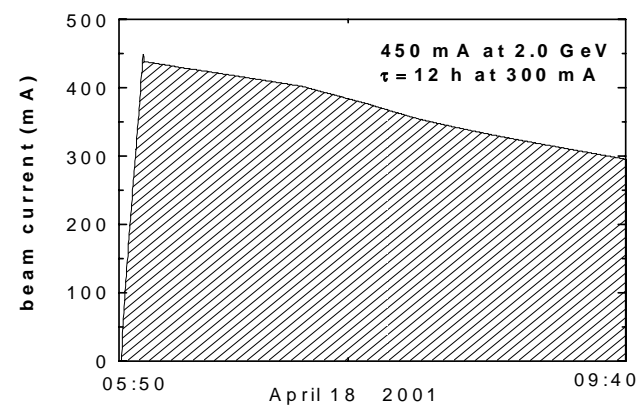

Fig. 1: Stored beam of $450 \mathrm{~mA}$ at $2.0 \mathrm{GeV}$. Different kind of beam instabilities excite as the beam current decays, which are indicated by discontinuities of the curve showing increasing or decreasing the beam loss.

Table 1: Beam-time and user service history

\begin{tabular}{|l|c|c|c|c|c|c|}
\hline & 995 & 696 & 97 & 698 & 69 & 2000 \\
\hline $\begin{array}{l}\text { Beam-time } \\
\text { provided(hr) }\end{array}$ & 1142 & 3034 & 3618 & 3784 & 3831 & 3884 \\
\hline $\begin{array}{l}\text { Proposals } \\
\text { submitted }\end{array}$ & 58 & 124 & 173 & 171 & 255 & 322 \\
\hline $\begin{array}{l}\text { Experiments } \\
\text { carried out }\end{array}$ & 18 & 69 & 139 & 130 & 156 & 237 \\
\hline $\begin{array}{l}\text { Number } \\
\text { of users }\end{array}$ & 78 & 283 & 577 & 646 & 659 & 883 \\
\hline
\end{tabular}

\section{MACHINE OPERATION IN YEAR 2000}

\subsection{Injector Linac}

The linear accelerator operated 5,280 hours in 2000, with a $1 \mathrm{~ns}$ pulse beam at $10 \mathrm{~Hz}$ repetition rate at the beam energy of $2.045 \mathrm{GeV}$. The operation time of the klystron and modulator system was 6,800 hours with the system availability of $94 \%$. The number of modulator faults that caused injection delays has decreased from 38 in 1999 to 29 in 2000 [3]. Overall, the operation of the linear accelerator was more stable than in previous years.

\subsection{Storage Ring}

The storage ring was operated for 4,834 hours in 2000 . The beam time scheduled to users was 4,224 hours, divided into 18 ten-day periods. The actual time supplied to users was 3,834 hours with a beam availability of $90.9 \%$. It is noteworthy that the beam energy was increased to $2.5 \mathrm{GeV}$. The average injection current was $166.9 \mathrm{~mA}$, and the average beam lifetime was 37.7 hours in 2000 [3]. 


\section{PROGRESSES IN THE MACHINE OPERATION}

After the last report on the PLS status [4], there have been fruitful progresses in PLS operation [3].

\subsection{Unification of the Control Room}

Two independent control rooms for $2 \mathrm{GeV}$ Linac and the storage ring were unified into a storage ring control room beginning from March 2000. After the merging of the control room, a period of the shift-duty has become doubled for machine operators. Also injection time is much saved by removing the need of telephone calls between two remote control operators for adjusting various injection parameters. Now the beam injection is possible by clicking the command buttons on a single console screen. Online help menus will be implemented for more secure and easier operation.

\subsection{Machine Operation at $2.5 \mathrm{GeV}$}

PLS is now regularly run at $2.5 \mathrm{GeV}$ with the stable energy ramping control system. The new energy ramping system [5] uses independent control system based on the Windows system for synchronization of all magnet controllers. With this high energy, x-ray flux increased and the beam stability is improved. No beam instability is observed at the present operational beam current of $180 \mathrm{~mA}$. Beam lifetime is also increased from 20 hours at $2.0 \mathrm{GeV}$ to 40 hours at $2.5 \mathrm{GeV}$ at $100 \mathrm{~mA}$. Shown in Fig. 2 is a beam current display for a successful ten-day operation.

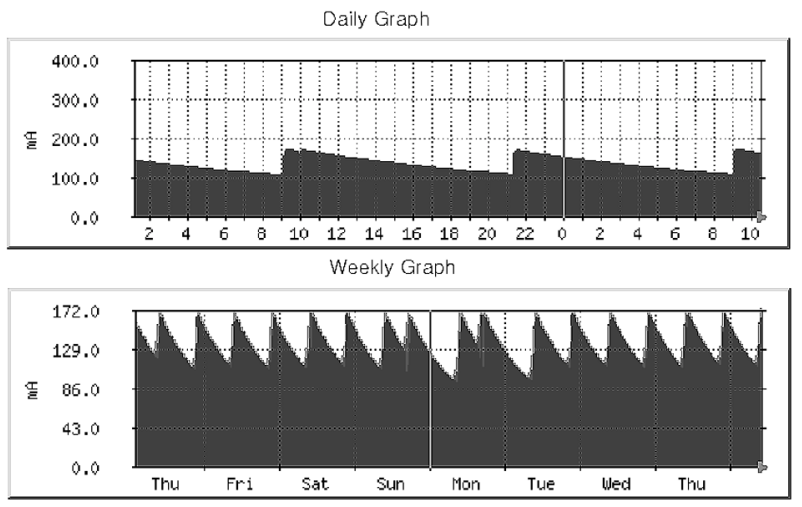

Fig.2: Beam current display for daily and weekly operation.

\subsection{Operation of the First Insertion Device U7}

From 2000, the first insertion device beamline U7 is successfully running for the user service. The orbit change with the change of U7 gap doesn't affect user experiment seriously yet. The second ID in PLS, EPU6, is installed and under commissioning now.

\subsection{Access to the Experimental Hall}

Experimental hall has been a restricted area during the beam injection until the end of 2000. This caused much inconvenience to the users because they must escape from their experimental stations. With a five-year accumulated radiation dose data for proof of the radiation safety, PLS finally obtained permission of access to the experimental hall during the beam injection from March 2001.

\subsection{Communication with Users}

For effective communications between user side and machine operation side, we made several understandings. Most important was on the maintenance of the orbit with a Reference Orbit and the strategy for realignment of the ground motion. Several information-broadcasting services are also provided for effective communications. Before the upgrade of the control system, we use a ftp server for machine data service to all staffs and users. There are three CATV channels for the broadcasting of the operation status and the pop-up messages. Also we utilize the web page [6] for information-broadcasting and pop-up messaging.

\section{ONGOING PROJECTS}

\subsection{Closed Orbit Stabilization}

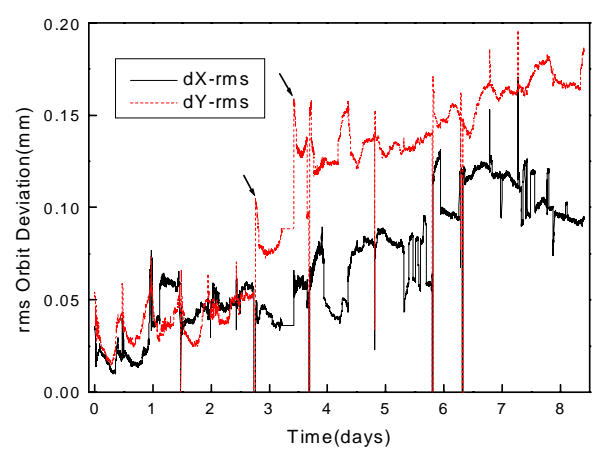

Fig. 3. RMS orbit deviation with operation time. Arrows indicate large increase of rms values by the degaussing of magnets by unexpected beam dumps.

In PLS long term orbit measurements show coherent drift of the orbit up to $+/-150 \mu \mathrm{m}$ as shown in Fig. 3. With careful investigations, we find that there is a strong correlation between the orbit drift and the temperature changes of the magnet and air in the storage ring tunnel. Major source of the temperature change is attributed to the large change of the magnet current during the regular beam injection: dump the stored beam, degauss the magnets with the full swing of the magnet current up to saturation, fill the beam at $2.0 \mathrm{GeV}$ and raise the energy again to $2.5 \mathrm{GeV}$. During this injection process, heat load changes around $200 \%$ of the normal operation. In contrary, when the machine is shutdown for several hours, it is over-cooled. Once heated up or cooled down, it takes around 4 hours for temperature to be stabilized in the vacuum chamber and supporting structures. To reduce the temperature shock, we applied de-ramping technique of the beam energy without dump the stored beam to refill. 
By this process, thermal shock on the magnets reduced significantly, and the change of the orbit before and after the injection is reduced significantly now. Further studies are still in progress [7].

\subsection{Upgrade of the RF System}

RF control electronics has made troubles making frequent machine trips. All the rf control electronics are thus redesigned and in fabrication now [3]. It will be finished at the end of 2001. Further, since the present $\mathrm{rf}$ power $(60 \mathrm{~kW} \times 4$ klystrons) will become short as the number of IDs increase as shown in Fig. 4, we will replace $60 \mathrm{~kW}$ klystrons with $75 \mathrm{~kW}$ ones one by one and add one more cavity making total rf power $375 \mathrm{~kW}$ without changing the waveguide system. Then we will have a capability of operation by 2005 at $200 \mathrm{~mA}$ with 10 IDs at $2.5 \mathrm{GeV}$ energy [8].

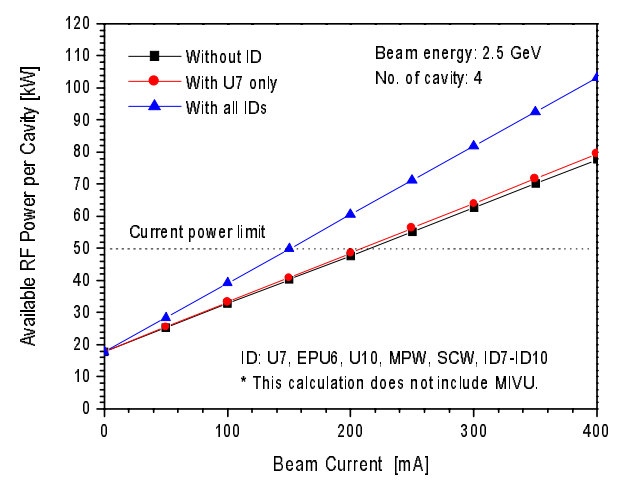

Fig. 4. Estimation of $\mathrm{rf}$ power for compensation of the radiation loss by operation of planned IDs.

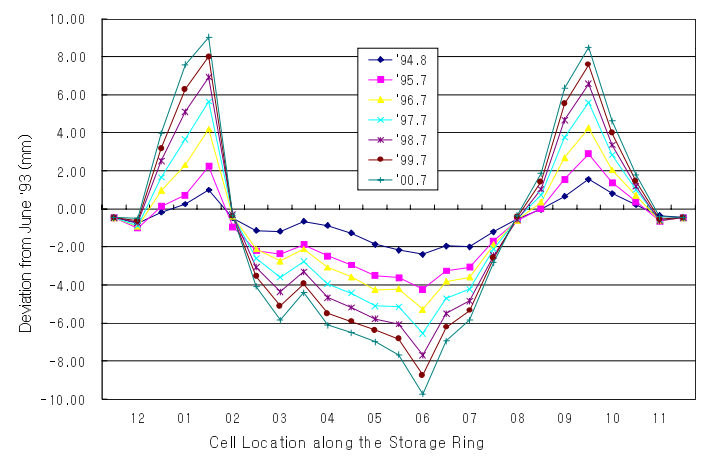

Fig. 5. Settlement of the storage ring floor after June 1993.

\subsection{Storage Ring Deformation and Realignment}

As shown in Fig. 5, the settlement of the storage ring tunnel continues at about $3.0 \mathrm{~mm}$ (hill to valley) per year in PLS. The total deformation has reached presently $19 \mathrm{~mm}$ compared to the reference elevation established in June 1993. Since the accumulated deformation still increases without slowdown, the smoothing technique [3] will not apply in the future. Thus we decided to realign all storage ring component toward the design orbit step by step in three years. Beamlines will also realign the elevation when it is necessary.

\subsection{Other Issues}

In beam diagnostics, there is a controversy in the estimation of the coupling coefficient between the one by the beam size measurement( $>7 \%)$ and the other one by the tune approach method(0.7\%) [9]. For accurate beam diagnostics, a new diagnostic station equipped with a precise beam size measurement system is under construction. Another important thermal problem is the error of beam position measurement from the BPMs caused by the distortion of the 10-m long single-piece chamber by the temperature change. Preliminary test showed a significant reduction of the error by cooling the vacuum chamber, suggesting that all the vacuum chambers should be cooled eventually. Finally, for the upgrade of the control system, we decided to apply EPICS for the PLS control system. Set up of the development system and the detailed proposal is in progress now. The new control system will be installed by the middle of 2003 .

\section{ACKNOWLEDGEMENTS}

Authors thank to all PLS staffs for their elaborations for effective maintenance of the accelerator components.

\section{REFERENCES}

[1] PLS Design Report (revised ed.), Pohang Accelerator Laboratory, 1992.

[2] E. S. Kim, et. al., "Limitation of Beam Current in the PLS Storage Ring," in this Proceedings.

[3] 1999/2000 Annual Report, Ed. C. W. Chung, PAL, 2001.

[4] J. Choi, et. al., "The Operational Status of PLS", Proceedings of PAC'99, New York, 1999.

[5] K. M. Ha, et. al., "Storage Ring Energy Ramping Control System," PAL Report, PAL-PUB 2001-006, 2001, written in Korean, mailto:hkm@ postech.ac.kr

[6] http://pal.postech.ac.kr/

[7] S. J. Park, J. Y. Huang, W. H. Hwang, and S. H. Nam, "Improvement of Orbit Stability Using PLS Beam Position Monitoring System," submitted to KAPRA Conference, Cheju, Korea, July 2001.

[8] H. S. Kang, "Estimation of Touschek Lifetime and RF Power for $2.5 \mathrm{GeV}$ Operation with Ten Insertion Devices," PAL Report, PAL-PUB 2000-013, 2000.

[9] E. S. Kim, et. al., "Correction of Horizontal and Vertical Coupling in the PLS," in this Proceedings. 